\title{
Research Paper \\ Study the Effect of Resilience Training on Reducing Stress and Communication Problems in the Primary Caregivers of the Elderly With Alzheimer Disease
}

\author{
Mahdi Ghezelsefloo ${ }^{1},{ }^{*}$ Nadereh Saadati $^{2}$, Zahra Yousefi $^{3}$, Malihe Zamanpour ${ }^{4}$
}

1. Department of Psychology, Faculty of Humanities and Sport Sciences, Gonbad Kavous University, Gonbad, Iran.

2. Department of Consulting, Faculty of Psychology and Educational Sciences, Pychology and Foreign Languages, Khorasgan (Isfahan) Branch, Islamic Azad University, Isfahan, Iran.

3. Department of Psychology, Faculty of Psychology and Educational Sciences, Pychology and Foreign Languages, Khorasgan (Isfahan) Branch, Islamic Azad University, Isfahan, Iran.

4. Department of Psychology, Faculty of Humanities, Arak Branch, Islamic Azad University, Arak, Iran

\begin{tabular}{l|l}
$\begin{array}{c}\text { Use yourdevice toscan } \\
\text { and read the artice online }\end{array}$ \\
attation: Ghezelsefloo M, Saadati N, Yousefi Z, Zamanpour M. [Study the Effect of Resilience Training on Reducing Stress \\
and Communication Problems in the Main Caregivers of the Elderly with Alzheimer Disease (Persian)]. Salmand: Iranian Journal \\
of Ageing. 2019; 14(3):284-297. https://doi.org/10.32598/sija.13.10.250
\end{tabular}

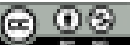

Received: 12 Apr 2019 Accepted: 25 Aug 2019 Available Online: 01 Oct 2019

Key words: Resilience, Stress, Communication problems, Caregiver

\section{A B S TRACT}

Objectives The caregivers of the family older member with Alzheimer disease face many challenges, sometimes describing as caring " 36 hours a day". This hard work affects various aspects of the caregivers' life, including health, occupation, and income. The current study was designed to determine the effectiveness of resilience training on reducing stress and communication problems of the primary caregivers of older people with Alzheimer disease.

Methods \& Materials The present research was an experimental study with a pre-test-post-test and a control group with two months follow-up. The samples were selected from the primary caregivers of the families who had received services from Tehran Hamraze Zendegi Center for elderly rehabilitation at home, under the supervision of the Welfare Organization, between September 23, 2017, and November 22, 2017. A sample size of 24 (Mean \pm SD: $73.20 \pm 9.09$ y) subjects were selected from 47 (Mean \pm SD: $42.41 \pm 7.51 \mathrm{y})$ older people with Alzheimer requesting services at home. Then, they were randomly divided into experimental and control groups. The data collection tools included "perceived stress scale" and "communication skills" questionnaire.

Results The variance analysis of repeated measurements showed that the resilience intervention was significantly effective in the experimental group in the post-test and follow-up stages (regarding stress and communication problems scores at the level of $\mathrm{P}=0.05$ ). The Bonferroni test results also indicated that the mean differences of stress and communication problems were significant between the pre-test with post-test and the follow-up stages $(P=0.0001)$. However, the mean difference between post-test and follow-up was not significant in any of the research variables, suggesting no return in the outcomes of the intervention at the follow-up stage. This finding confirmed the sustainability of the intervention effect.

Conclusion Considering older people's need to be supported by their relatives, resilience training appears to be an effective method capable of reducing stress and communication problems in caregivers of older people with Alzheimer disease.

\section{* Corresponding Author:}

Nadereh Saadati, PhD.

Address: Department of Consulting, Faculty of Psychology and Educational Sciences, Pychology and Foreign Languages, Khorasgan (Isfahan Branch, Islamic Azad University, Isfahan, Iran.

Tel: +98 (913)1253203

E-mail: n.saadati@khuisf.ac.ir 


\section{Extended Abstract}

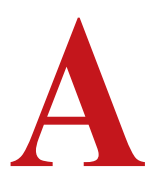

\section{Introduction}

long with the rise in the proportion of older people in most countries, the number of people with dementia also increases [3], which causes serious damages to individuals, society, and the economy as a global health problem [4]. Owing to its serious consequences for the patient and the family, Alzheimer disease is one of the most worrisome disorders at present, described as "endless funerals" [8]. Because of the consequences and complications of Alzheimer disease, like psychological and psychiatric changes along with the disruption of daily living activities, memory impairment, and other cognitive functions (judgment and thinking, etc.), the caregivers should assist and supervise these patients in their daily activities [14]. This study aimed to investigate the effectiveness of resilience training in reducing stress and communication problems in the primary caregivers of older people with Alzheimer disease.

\section{Materials and Methods}

This research is a quasi-experimental study with the pre-test, post-test design using a control group with a tow month follow-up period. The study population consisted of all primary caregivers (spouses, children, and daughtersin-law) of families, who had benefited from rehabilitation services in Hamraz-E Zendegi Nursing Home in 2017. For sampling, we used the convenience sampling method. The caregivers complied with the principles of confidentiality and cooperated with consent. They had no history of illness or treated by medication and psychotherapy. Then, we asked the primary caregivers of 47 older people with Alzheimer's, who requested home services (medicine, nursing, occupational therapy, etc.) from the nursing home, to

A

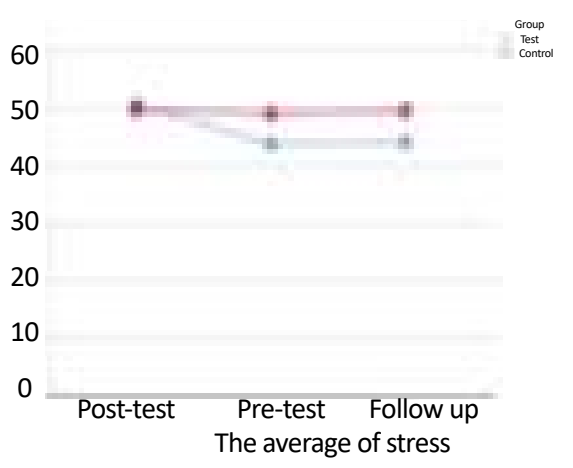

Figure 1. Interactive charts of resilience training effectiveness complete the Perceived Stress Scale (PSS) developed by Cohen et al. [32] and the Communicative Problems Survey (CPS) designed by Khatooni and Zohari [10]. After initial screening, 29 subjects earned high PSS and CPS scores, 24 of whom were selected according to the inclusion criteria and were randomly assigned to training $(\mathrm{n}=12)$ and control $(\mathrm{n}=12)$ groups.

The training group received 8 group sessions of resilience training for one month, tow sessions per week, each taking 90 minutes. At the end of the treatment sessions and after tow months, a follow-up was performed for both groups. Then, the data of three stages of pre-test, post-test, and follow-up in both groups were analyzed in SPSS, using Levene's test, Kolmogorov-Smirnov test, Chi-squared test, and repeated measures ANOVA at a significance level of less than 0.05 .

\section{Results}

Among caregivers in the training group, $3(25 \%)$ were spouses, $7(58.3 \%)$ were children, and $2(16.7 \%)$ were daughters-in-law of the older people. In the control group, tow caregivers $(16.7 \%)$ were spouses, 7 (58.3\%) were children, and 3 (25\%) were daughters-in-law. In terms of education in the training group, 2 caregivers had a high school diploma, 1 had an associate degree, 7 had a bachelor's degree, and 2 had a master's degree. In the control group, 3 had an associate degree, 7 had a bachelor's degree, and 2 had a master's degree. The Mean \pm SD age of the participants was $43.33 \pm 5.85$ years in the training group and $41.50 \pm 9.05$ years in the control group.

The results presented in Table 1 show a significant reduction in stress and communicative problems in the training group compared to the control group at post-test and follow-up period. Figure 1 compares the mean val-

B

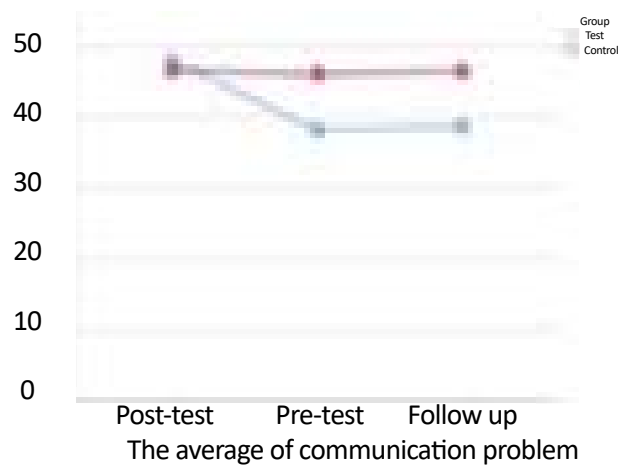

A. Comparing the mean scores of stress; B. Communicative problems 
Table 1. Stress and communicative problems in both groups at three measurement stages

\begin{tabular}{ccccc}
\hline \multirow{2}{*}{ Variable } & Group & \multicolumn{3}{c}{ Mean \pm SD } \\
\cline { 3 - 5 } & & Pre-test & Post-test & Follow-up \\
\hline \multirow{2}{*}{ Stress } & Training & $48.00 \pm 3.83$ & $38.18 \pm 4.48$ & $38.70 \pm 4.27$ \\
& Control & $46.58 \pm 4.50$ & $46.08 \pm 4.31$ & $46.50 \pm 4.21$ \\
Communicative problems & Training & $50.58 \pm 3.65$ & $43.75 \pm 3.93$ & $44.00 \pm 3.41$ \\
& Control & $49.66 \pm 4.09$ & $49.00 \pm 4.11$ & $49.50 \pm 4.01$ \\
\hline
\end{tabular}

ues of stress and communicative problems in both groups. Based on the results of repeated measures ANOVA, the difference in the mean scores of stress and communicative problems between study groups at post-test and the follow-up period was significant $(\mathrm{P}<0.05)$. Also, the results of the Bonferroni post hoc test showed a significant difference between the pre-test mean scores of stress and communication problems and those of post-test and follow-up scores $(\mathrm{P}=0.0001)$. However, the mean difference between post-test and follow-up scores was not significant in any of the research variables, indicating that the effect of resilience training was stable in our study.

\section{Conclusion}

As the caregivers of older people with Alzheimer disease experience stress due to elderly care, proper strategies should be used to improve the physical and mental health of these people. The resilience training program, in other words, a multidimensional approach that affects the thoughts, emotions, and behaviors of individuals and caregivers, can have a significant impact on reducing stress and enhancing communication skills. One of the limitations of the present study was the small size of the study subjects. Thus, we suggest that more samples be selected in future studies. Moreover, the present study was conducted in Tehran, and the results cannot be generalized to other cities of Iran. Hence, it is suggested that similar studies be conducted in other cities.

\section{Ethical Considerations}

Compliance with ethical guidelines

This study was approved by the Research Ethics Committee of Gonbad Kavous University and Deputy for Research of the Department of Welfare and Rehabilitation Affairs in Tehran, Iran.

Funding

This article was extracted from a research proposal approved by the Gonbad Kavous University and received financial support from the Deputy for Research of the Department of Welfare and Rehabilitation Affairs in Tehran, Iran.

\section{Authors' contributions}

Conceptualization by Maliheh Zamanpour and Mehdi Ghezelsefloo; Methodology and validation by Nadereh Saadati, Zahra Yousefi and Mehdi Ghezelsefloo; Investigation by Nadereh Saadati and Zahra Yousefi; Editing and final draft preparation by Nadereh Saadati and Mehdi Ghezelsefloo.

\section{Conflicts of interest}

The authors declared no conflict of interest. 


\title{
بررسى اثربخشى آموزش تابآورى بر كاهش استرس و مشكلات ارتباطى در مراقبتكنيندكان اصلى سالمندان مبتلابه آلزايمر شهر تهران بران
}

\author{
مهدى قزلسفلو' ، "نادره سعادتى' ، زهرا يوسفى"، مليحه زمان يور” \\ ا- كروه روانشناسى، دانشكده علوم انسانى و علوم ورزشي، دانشكاه كنبد كاووس، كنبد، ايران.

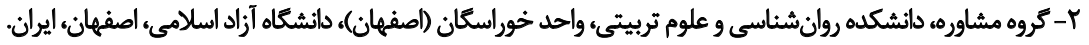

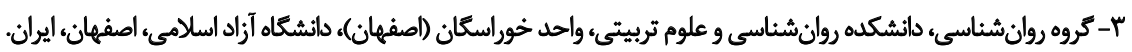

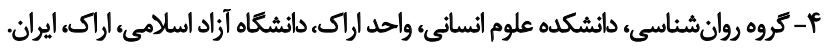

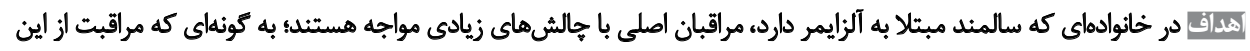

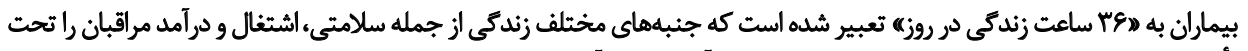

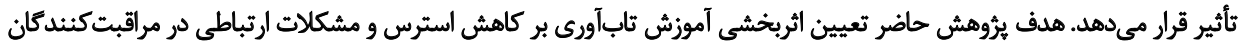
الصلى سالمثدان مبتلابه آلزايمر بود.

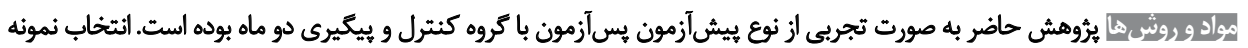

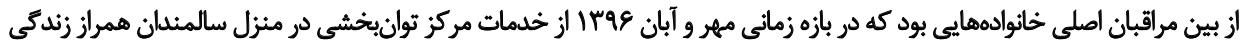

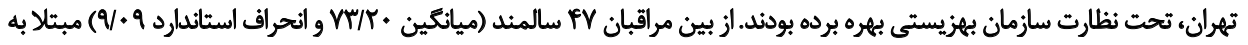

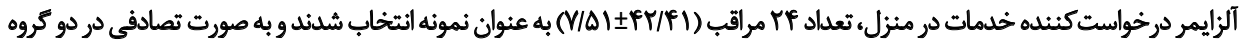

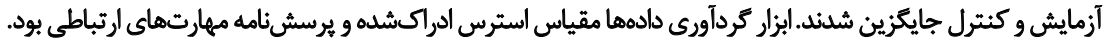

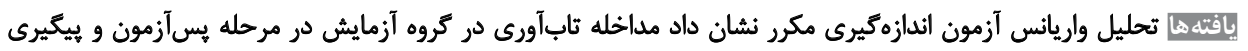

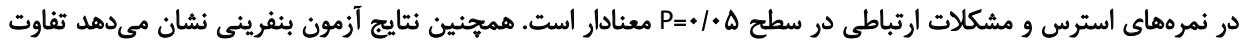

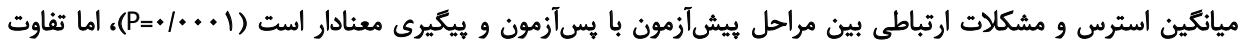

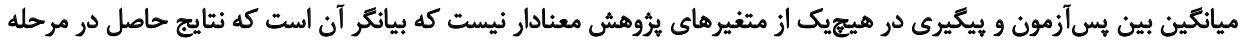

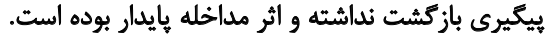

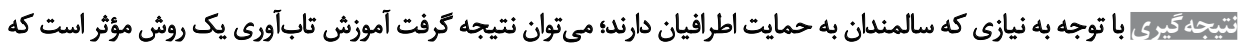

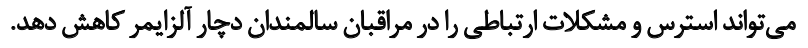

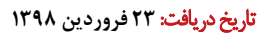
تاريخ هذيرش: بـ شهريور

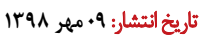

عقل، نوعى اختلال مزمن، يِيجيده و وييشرونده عصبي است كه

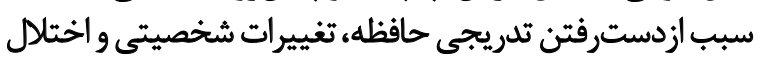

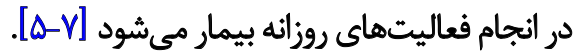

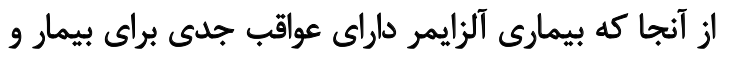

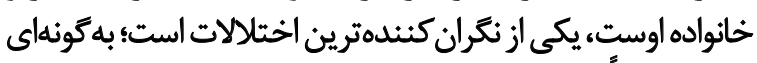

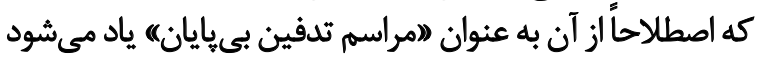

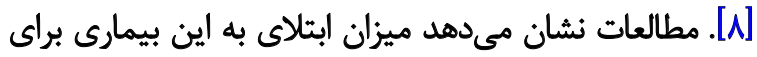

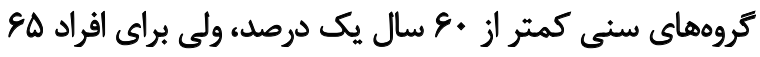

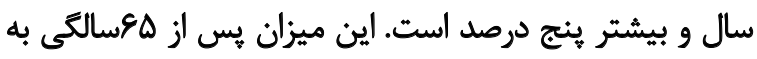

سالمندى مرحلهاى از سير طبيعى زندگى انسان است كه براى

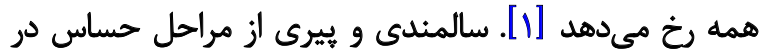

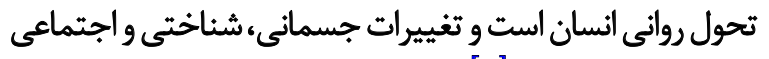

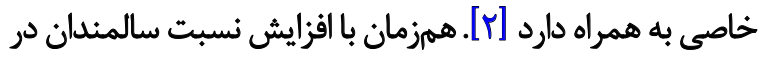

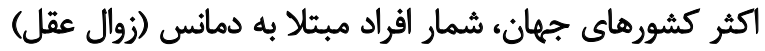

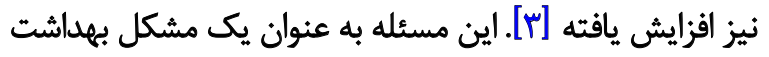

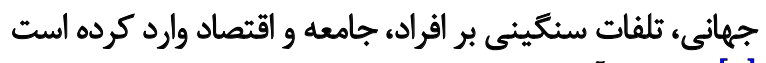

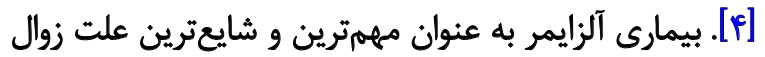

두. 
ميانى برقرارى مصاحبه به مدت طولاتى و نيز خواندن مطلب

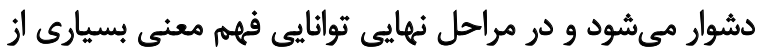

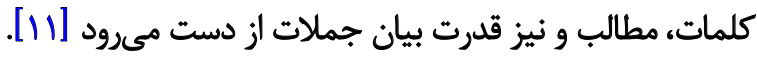

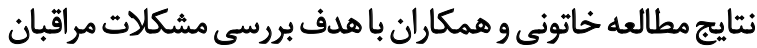

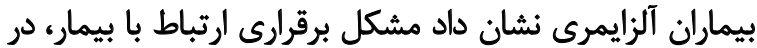

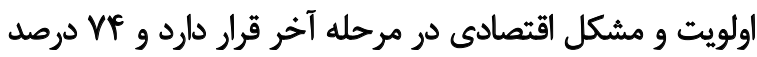

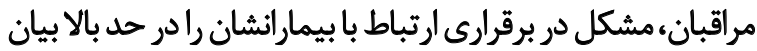

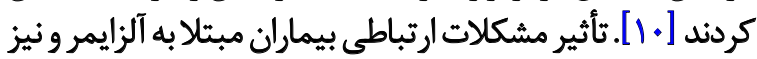

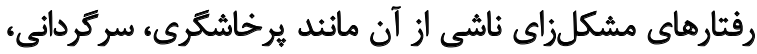

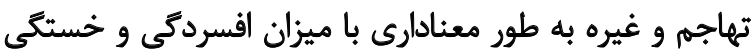

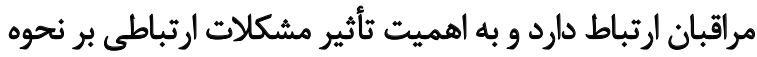

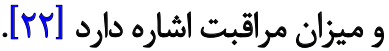

با درنظركرفتن اين حقيقت كه استرس بالاى مراقبان بيماران

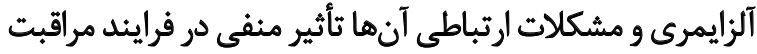

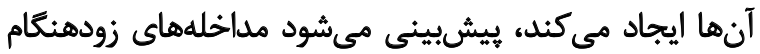

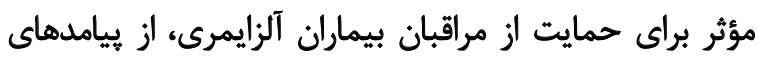

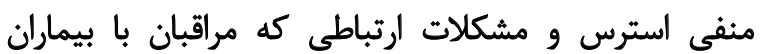

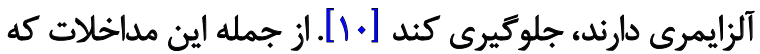

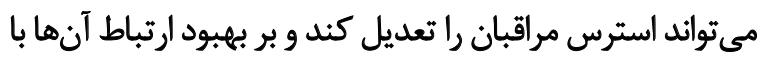

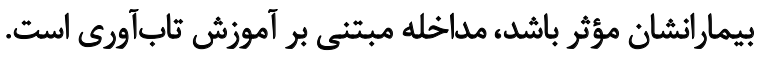

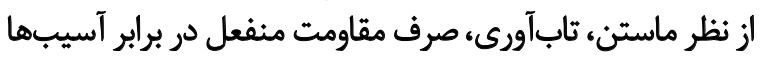

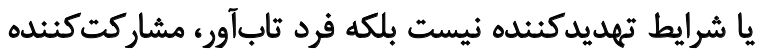

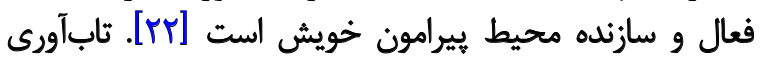

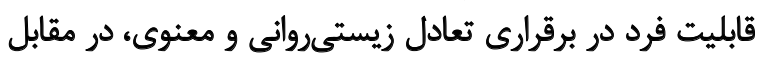

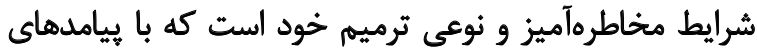

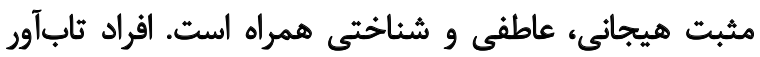

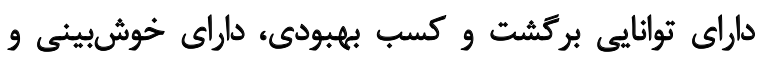

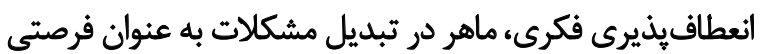

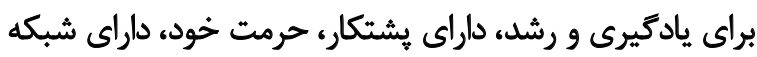

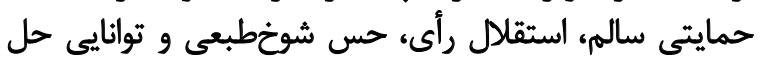

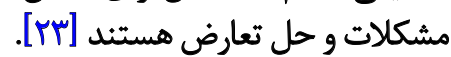

ثابآورى به عنوان توانايى جيرهشدن بر استرس و كرفتارى

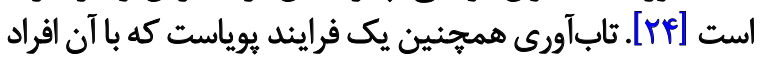

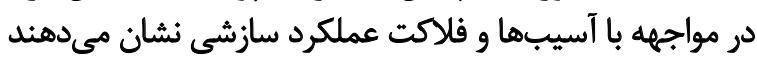

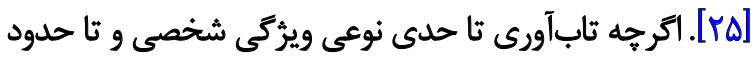

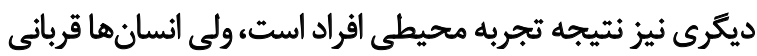

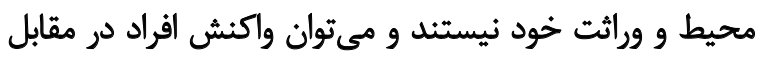

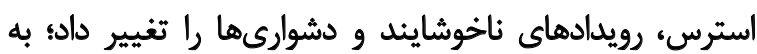

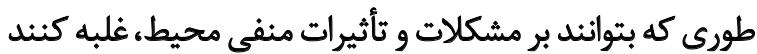

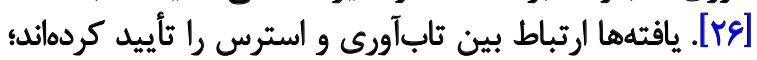

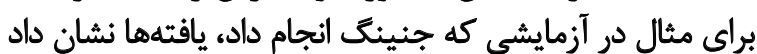

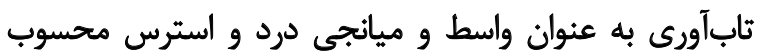

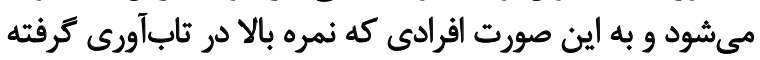

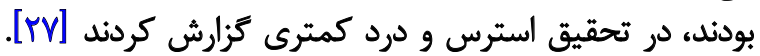

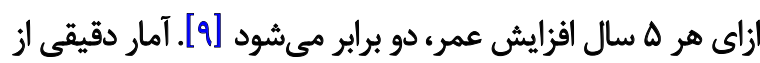

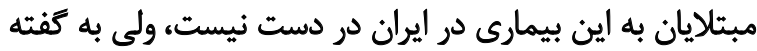

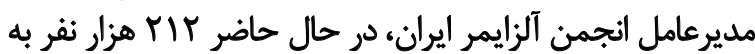

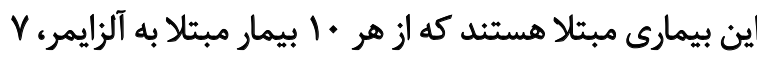

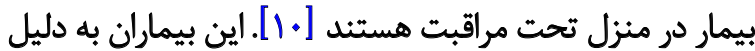

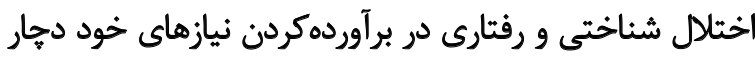

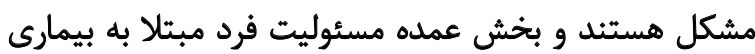

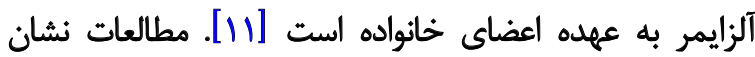

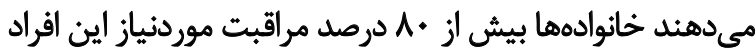
را تأمين مي كنيند [IT)

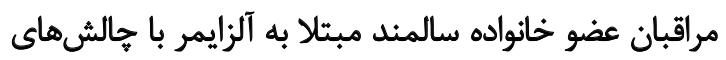

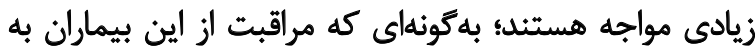

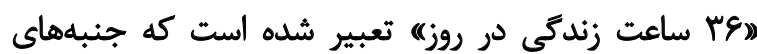

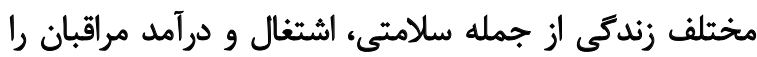

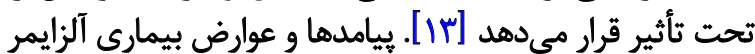

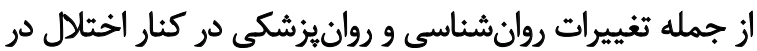

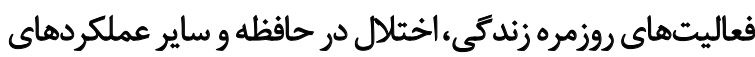

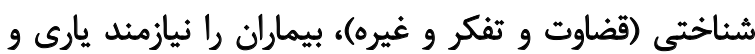

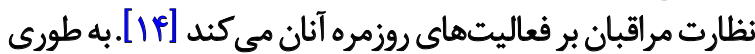

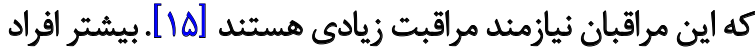

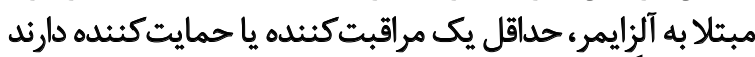
كه معمولاً همسر يا خويشاوندان بيمار هستئد [19].

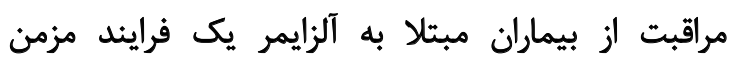

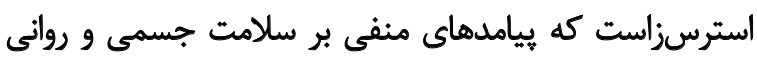

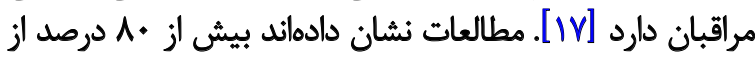

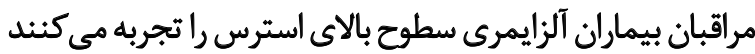

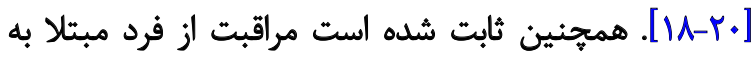

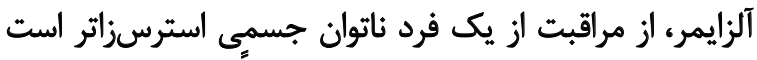

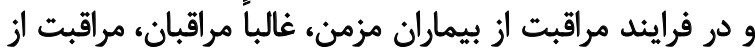

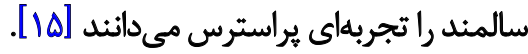

از سوى ديكر مشكلات ارتباطى در بيماران آلزايمرى بسيار

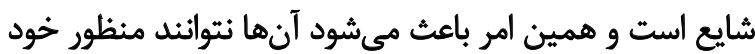

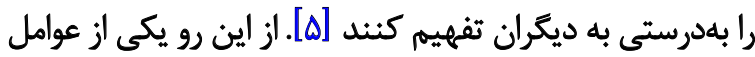

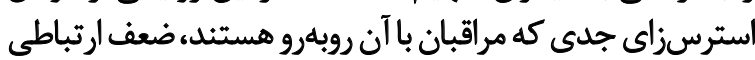

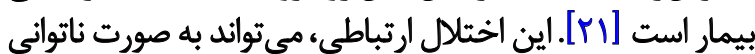

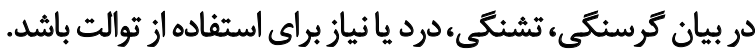

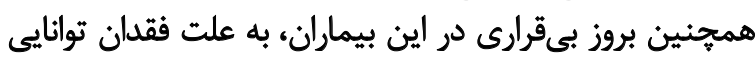

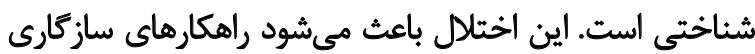
كاهش يابند و احتمال تنش قوت بكيرد [هاء].

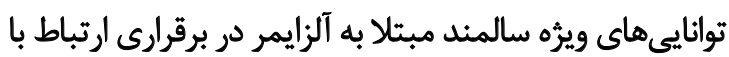

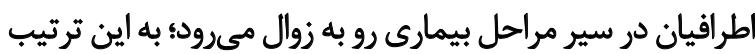
كه در مراحل اوليه، تمركز براي بيمار سخت مي بـوده، در مراحل 
هفتهاى دو جلسه آموزش تابآورى اجرا شد. در اين بروهش

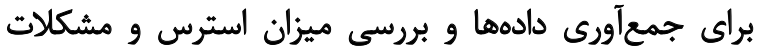

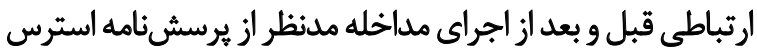

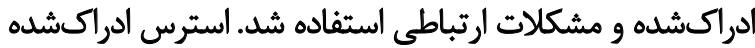

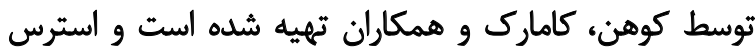

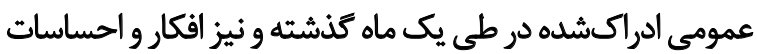

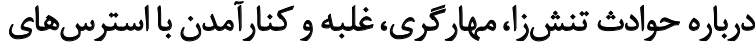

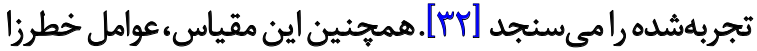

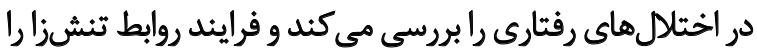

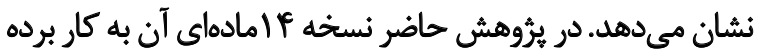

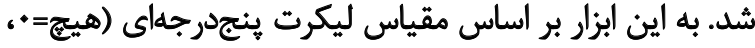

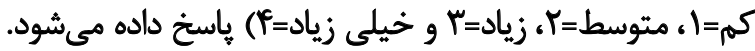

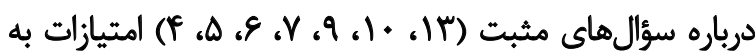

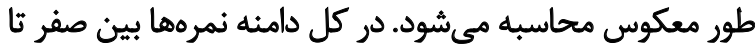

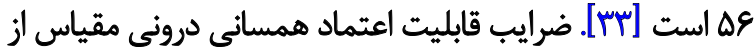

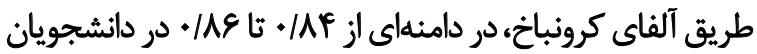

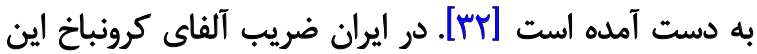

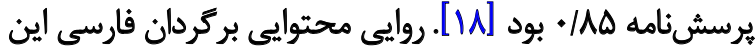

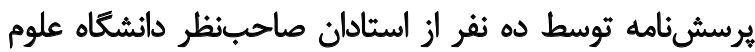

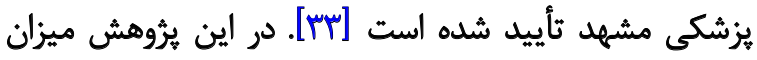

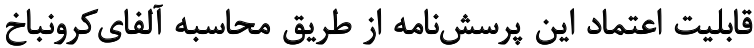

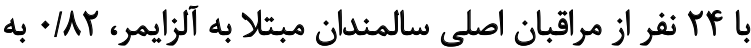

دست آمده است.

همجنين خاتونى و زهرى يرسشنامه مشكلات ارتباطى در

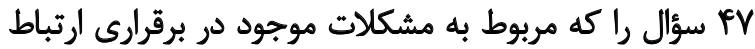

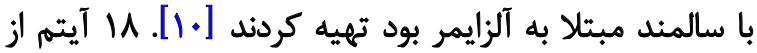
بخش اخير، منعكس كننده مشكلات مراقبت كنينده در فهميدان

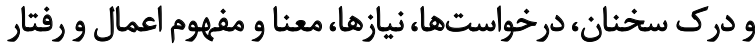

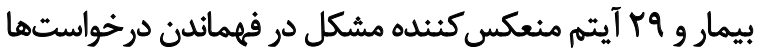

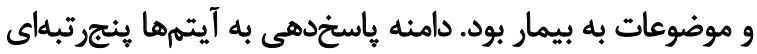

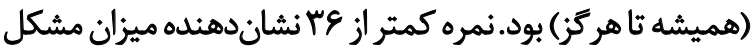

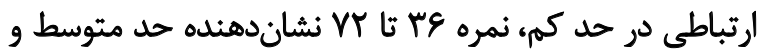

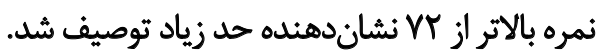

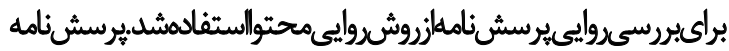

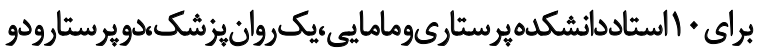

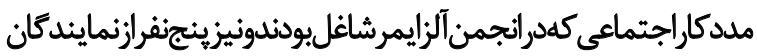

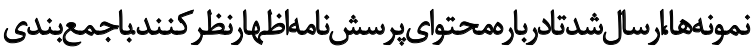

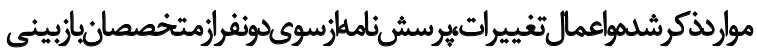

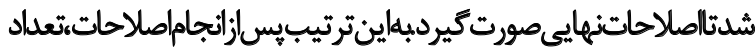

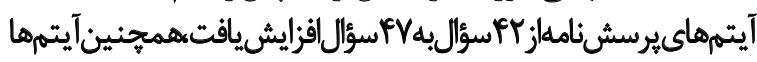

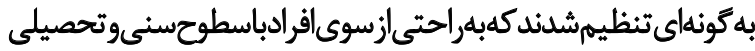

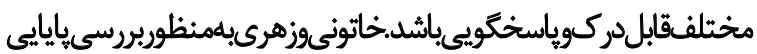

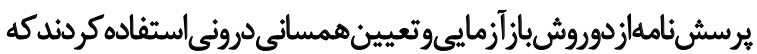

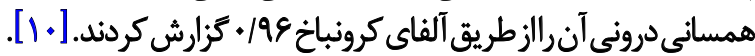

همجينين آموزش تابآورى اين امكان را به مراقبان بيماران

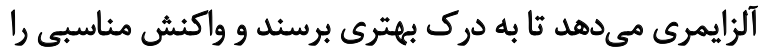

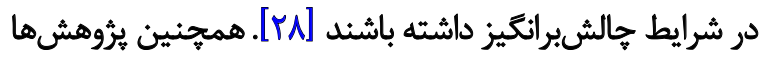

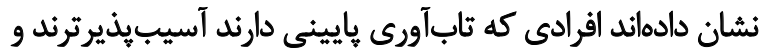

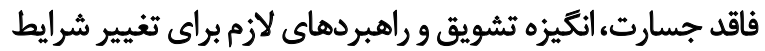

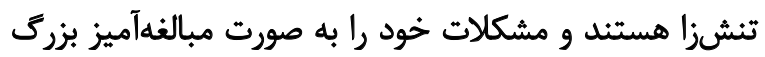

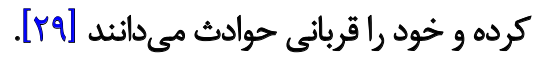

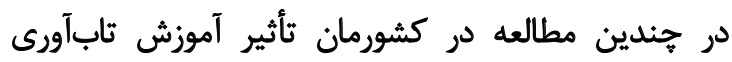

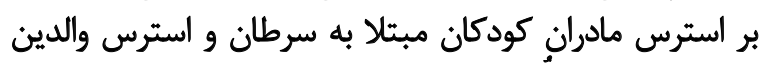

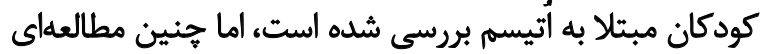

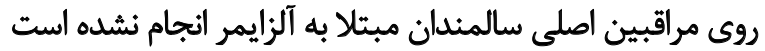

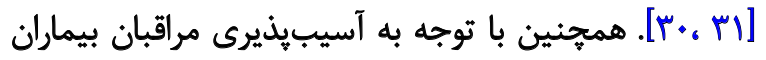

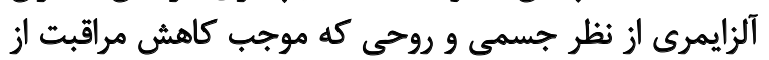

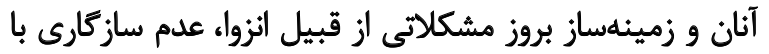

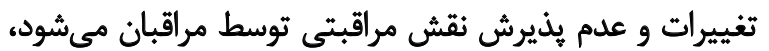

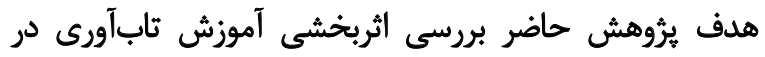

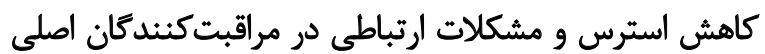
سالمندان مبتلا به آلزايمر است. روش مطالعه اين يرُوهش از طرحهاى تجربى و كاربردى است كه به صورت

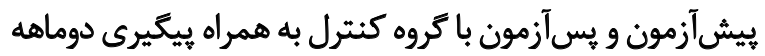

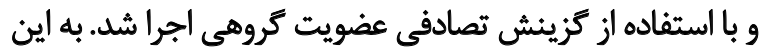

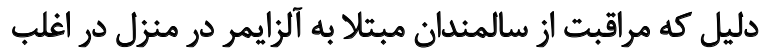

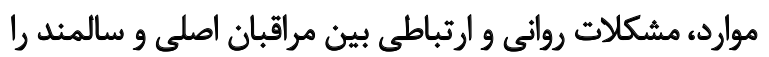

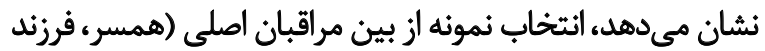

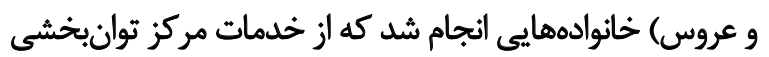

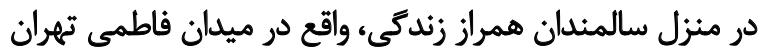

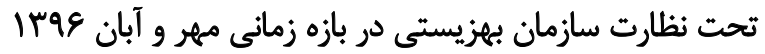

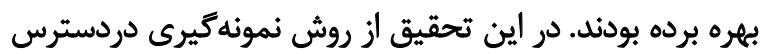

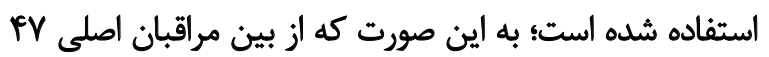

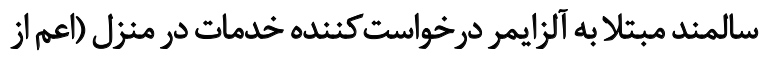

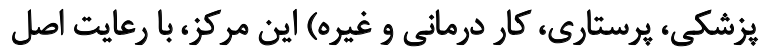

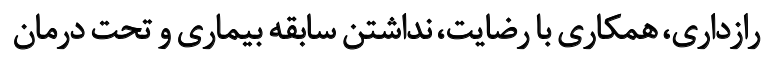

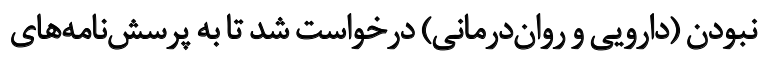

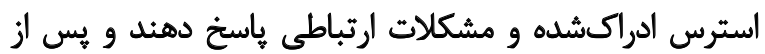

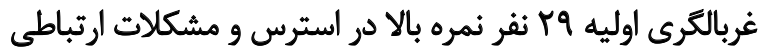

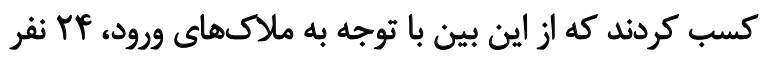

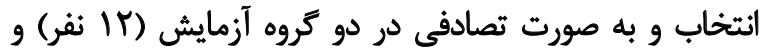
كنترل (rا نفر) جايكزين شدند.

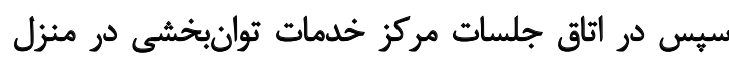

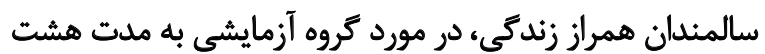

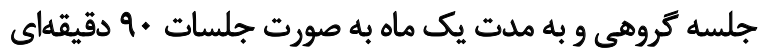


مسئوليتيذيرى فردى و بذيرش نقشهاى معنادار)، آشنايى

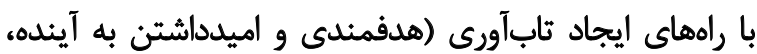

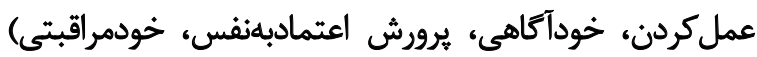

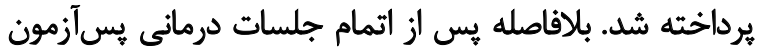

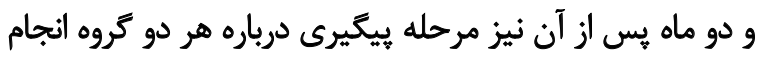

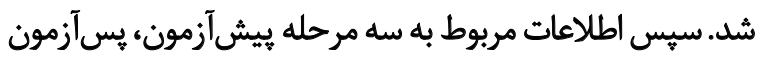

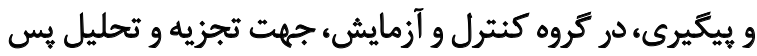

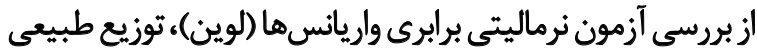

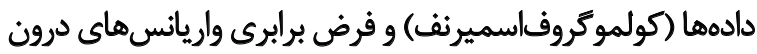

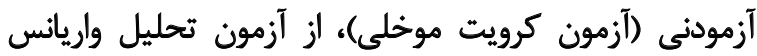

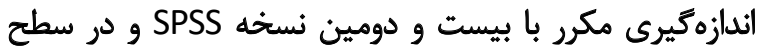
Pنادارى

يافتهها

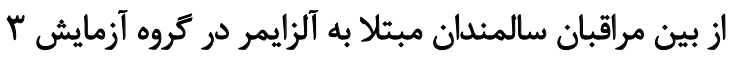

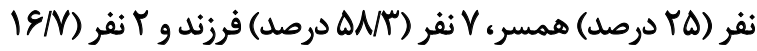

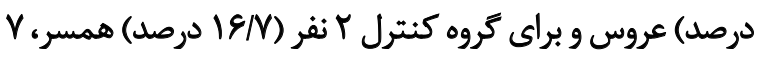

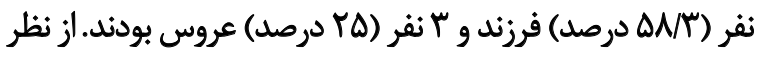

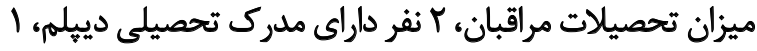

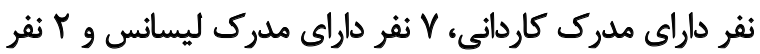

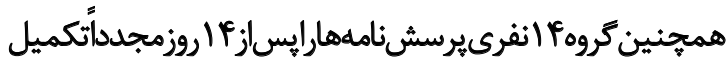

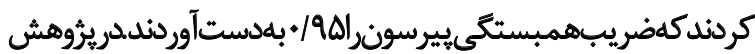

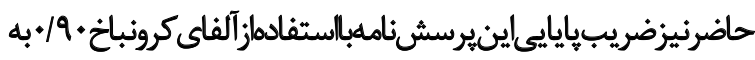

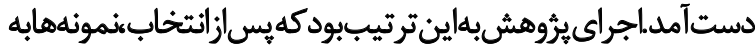

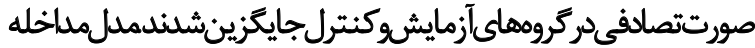

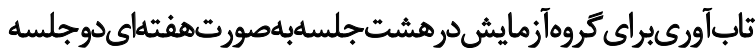

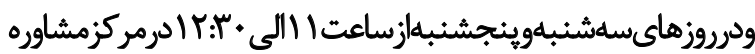

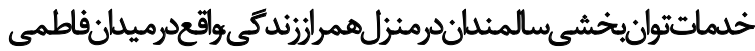
تهراناجر اشدوبهمدت

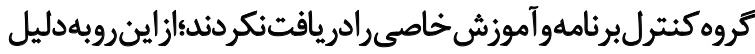

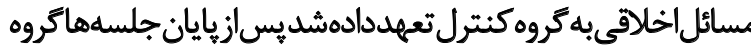
آزمايش، آنها نيز تحت مداخله قرار كرفيتند.

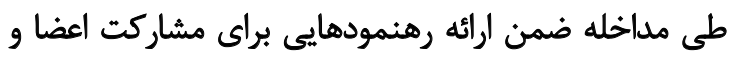

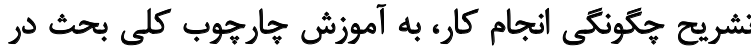

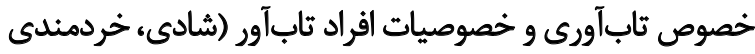

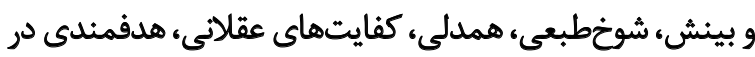

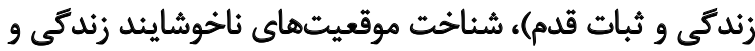

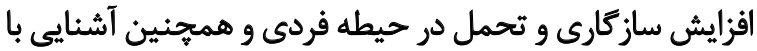

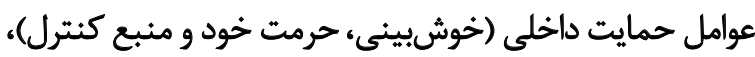
آشنايى با عوامل حمايت خارجى (سيستم حمايت اجتماعي،

جدول 1. اطلاعات جمعيتشئاختى مراقبت كنئدكان اصلى از سالمندان مبتلا به آلزايمر

\begin{tabular}{|c|c|c|}
\hline تعداد (درصد) & \multicolumn{2}{|c|}{ متغير } \\
\hline $10(8 T / \Delta)$ & زن & \multirow{2}{*}{ جنس } \\
\hline $9(N V / \Delta)$ & مرد ل مرد & \\
\hline$P(I E N)$ & 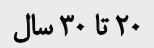 & \multirow{3}{*}{ سن سن } \\
\hline$r(I r / \Delta)$ & 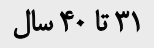 & \\
\hline $\operatorname{IV}(V \cdot / A)$ & أf سال به بالا & \\
\hline$\Delta(r \cdot 1 A)$ & لهمبر & \multirow{3}{*}{ نسبت مراقب با بيمار } \\
\hline If $\left.(\Delta N)^{*}\right)$ & فرزئد & \\
\hline$\Delta(r \cdot / A)$ & عروس & \\
\hline $\operatorname{IV}(V+/ \Lambda)$ & متأهل & \multirow{2}{*}{ وضعيت تأهل } \\
\hline$V(r q / r)$ & مجرد & \\
\hline$r(\mathcal{M} M)$ & دييلم & \multirow{4}{*}{ ميزان تحصيلات } \\
\hline$F(I E N)$ & فوقدييلم & \\
\hline If $(\Delta N T)$ & ليسائس & \\
\hline$P(I E M)$ & فوقليسائس & \\
\hline$P(I E M)$ & شاغل & \multirow{3}{*}{ وضسيت الشتغال } \\
\hline Ir (AP/T) & خانهدار & \\
\hline$V(r q / T)$ & بيكار & \\
\hline
\end{tabular}


جدول r. دادهاي توصيفى كروه آزمايش و كنترل در مراحل بيش آزمونه يسآزمون و بيكيرى

\begin{tabular}{|c|c|c|c|c|}
\hline \multicolumn{3}{|c|}{ ميانكين土|نحراف معيار } & \multirow{2}{*}{ كوروه } & \multirow{2}{*}{ متغيرها } \\
\hline بيئيرى & يسآزمون & بيشآزمون & & \\
\hline$r N V+ \pm P / T V$ & YNMAER/PA & $P N \cdot . \pm r / A r$ & آزمايش & \multirow[b]{2}{*}{ استرس } \\
\hline$P \& / \Delta . \pm f / M)$ & $\varphi \& / \cdot A \pm \varphi / M)$ & $\varphi g / \Delta \Lambda+\varphi / \Delta$. & كترل & \\
\hline$p \varphi / \cdot . \pm r / p l$ & $P \Psi / V \Delta \pm \Psi / Q \Psi$ & $\Delta \cdot / \Delta \Lambda \pm \Psi / \varepsilon \Delta$ & آزمايش & \multirow{2}{*}{ شكلات ارتباطى } \\
\hline$F q / \Delta . \pm f / \cdot 1$ & $\mid+q / \cdots \pm f / 11$ & $F q / F \notin \pm F / . q$ & كنترل & \\
\hline
\end{tabular}

بر اساس نتايج جدول شماره F، سطح معنادارى تحليل واريانس

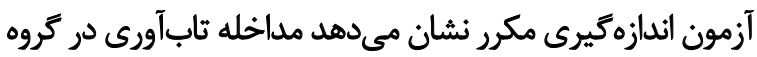

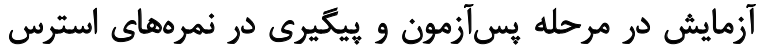

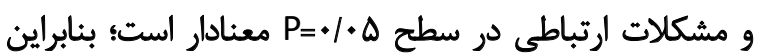

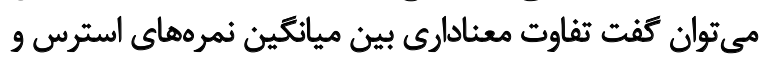

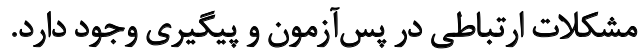
در جدول شماره ه و تصوير شماره ا نتايج آزمون بنفرينى نشان

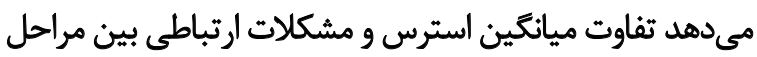

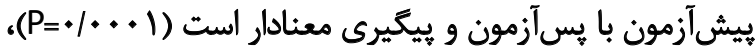

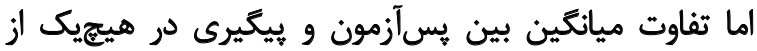

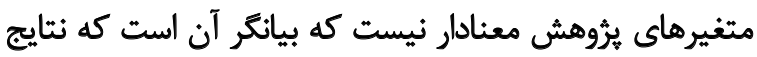

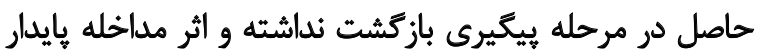

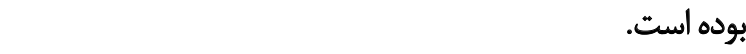

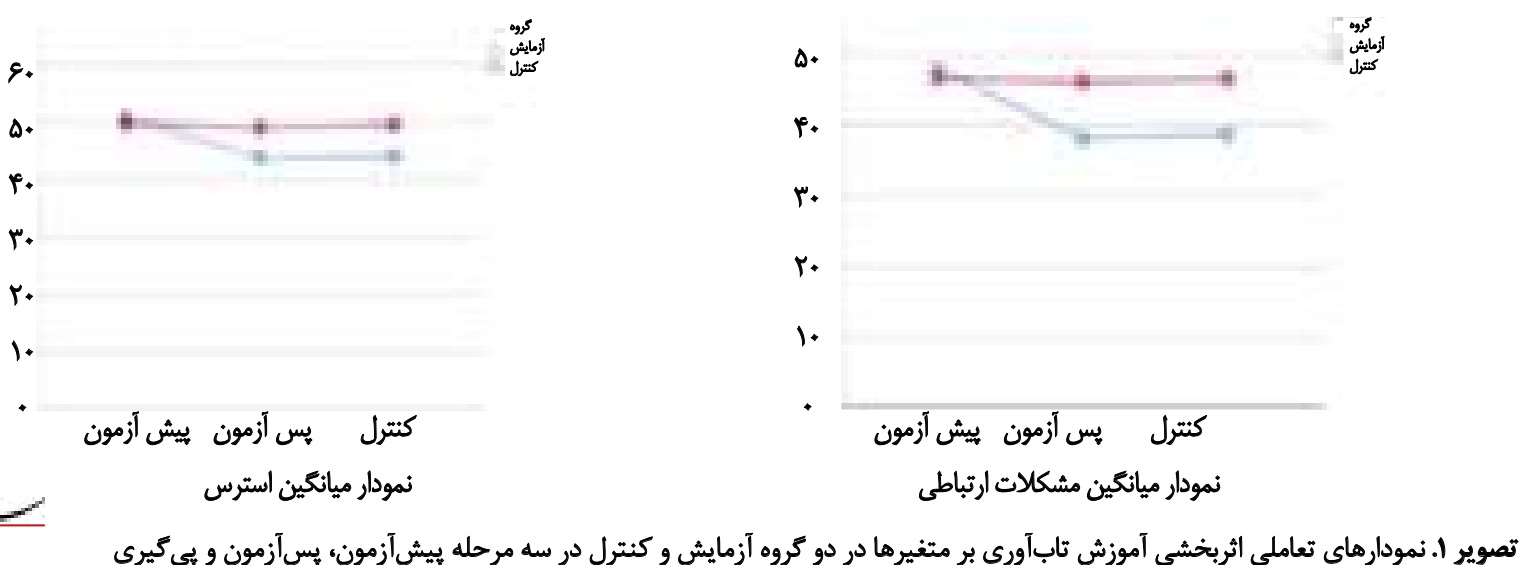

جدول r. خلاصه نتايج آزمون همكنى واريائسهاى استرس و مشكلات ارتباطى

\begin{tabular}{|c|c|c|c|c|c|c|}
\hline \multicolumn{2}{|c|}{ W موخلى W } & \multicolumn{2}{|c|}{ كولموكر افساسميرنف } & \multicolumn{2}{|c|}{ لوين } & \multirow{2}{*}{ متغير } \\
\hline آماره & 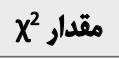 & معنادارى & آماره & معنادارى & آماره & \\
\hline .119 & T &.$/ 8 \mathrm{~A}$ & $\cdot M$ & $\cdot / \Delta$ &.$/ 48$ & استرس است \\
\hline ( & $1 / 48$ & ./94 & / $/ \Delta r$ &.$/ 8 \pi$ & . & مشكلات ارتباطي \\
\hline
\end{tabular}

داراى مدرك فوق ليسانس بودند و مراقبان كروه كنترل نيز ب نفر

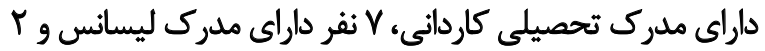

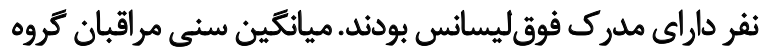

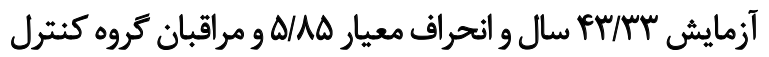

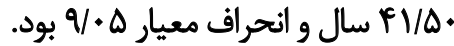

اطلاعات كلى جمعيتشناختى براى هر دو كروه در جدول

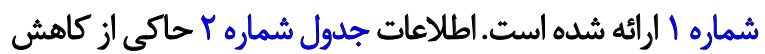

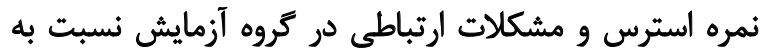

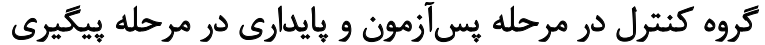

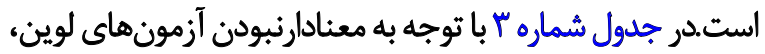

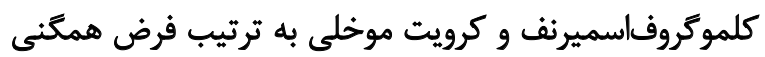

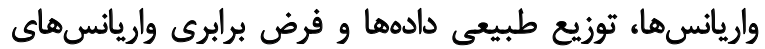

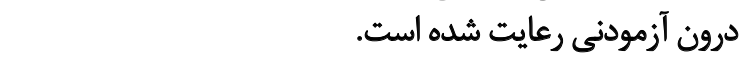

$$
\text { نمودار ميانكين مشكلات ارتباطي كترل }
$$

تصوير ا. نمودارهاى تعاملى اثربخشى آموزش تابآورى بر متغيرها در دو كزوه آزمايش و كنترل در سه مرحله بيش آزمون، بِ آزمون و يبى<smiles>C1CCCCC1</smiles> 
جدول F. نتايج تحليل اندازهيرى مكرر در سه مرحله ييشآزمون، يَسآزمون و ييكيرى

\begin{tabular}{|c|c|c|c|c|c|c|c|}
\hline ضاثريب & معنادارى & $F$ & مجذذورات & آزادى & مجموع مربعات & منيع & متغير \\
\hline \multirow{2}{*}{. /TQ } & \multirow{2}{*}{.1 .1} & \multirow{2}{*}{ V/gA } & $F \cdot V / \Delta \Delta$ & 1 & $P \cdot V / \Delta \Delta$ & اثر آموزش & \multirow[b]{2}{*}{ استرس } \\
\hline & & & $\Delta \% \% \varnothing$ & $\pi$ & $11 \mathrm{gV} / \mathrm{Mf}$ & خطا & \\
\hline \multirow{2}{*}{.118} & \multirow{2}{*}{$1+r$} & \multirow{2}{*}{ t/f. } & $19 \% / r A$ & 1 & $19 \pi / 41$ & اثر آموزش & \multirow{2}{*}{ مشكلات ارتباطى } \\
\hline & & & $P r / q r$ & rr & qse/W & خط & \\
\hline
\end{tabular}

جدول ه. نتايج آزمون تعقيبى بنفرونى در سه مرحله بيش آزمون، بس آزمون و بيكيرى

\begin{tabular}{|c|c|c|c|c|c|c|c|c|c|}
\hline \multicolumn{3}{|c|}{ هس آزمون-ييكيرى } & \multicolumn{3}{|c|}{ ييش آزمون-ييكيرى } & \multicolumn{3}{|c|}{ يبشأزمون-يس أزمون } & \multirow[b]{2}{*}{ شاخصهاى آمارى } \\
\hline معنادارى & انحراف & تفياتكين & معنادارى & انحراف & ثفانفين & معنادارى & انحراف & مياتكين & \\
\hline $.1 .+1$ & . & P/9A & $.1 .+1$ &.$/ M \varphi$ & $\Delta / 1 \Delta$ &.$/ \pi r$ &.$/ 10$ &.$/ 9 V$ & استرس \\
\hline $.1 .+1$ &.$/ Y F$ & $r / T r$ & $.1 . .1$ &.$|r|$ & $r / N \Delta$ & . $/ \mathrm{r}$ &.$/ r$. &.$/ \pi r$ & مشكلات ارتباطى \\
\hline
\end{tabular}

روى بهبود ارتباط با ديكران مؤثر است و نتايج رُّين و همكاران

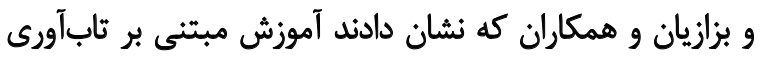

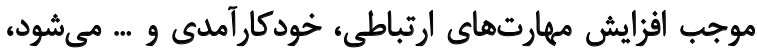

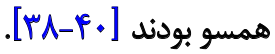

در تبيين اين يافتهها مي توان كفت تابآورى با افزايش عواطف

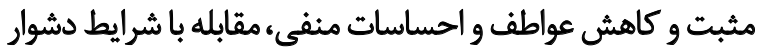

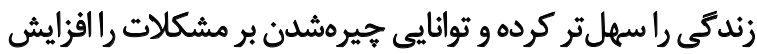

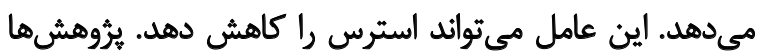

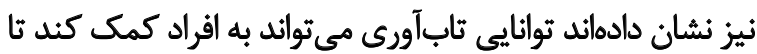

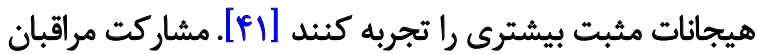

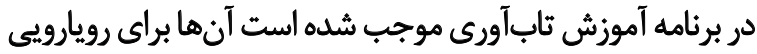

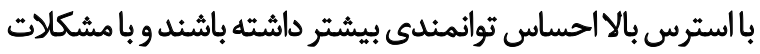

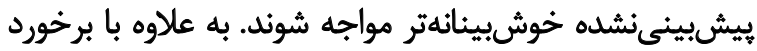

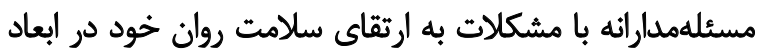

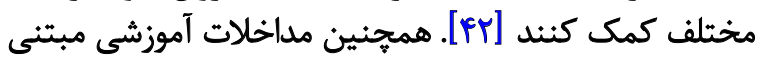

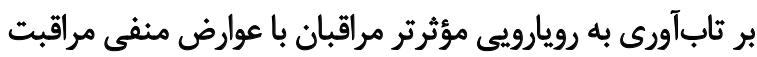

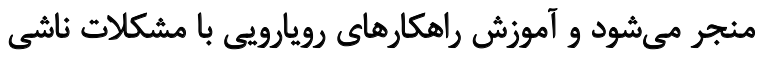

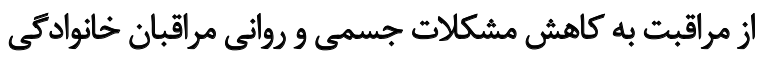

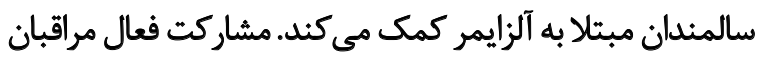

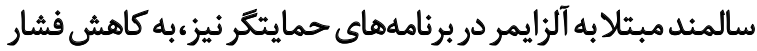

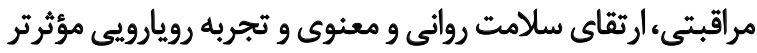

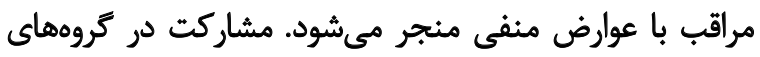

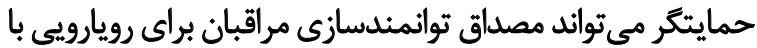

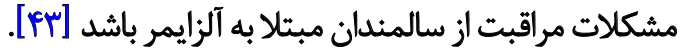
مراقبان با تابآورى بالاتر به طور مؤثرترى قادر به استفاده از

هدف برُوهش حاضر بررسى اثربخشى آموزش تاب آورى بر كاهش

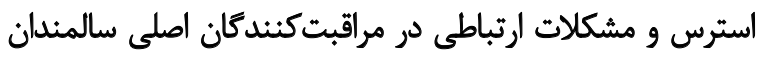

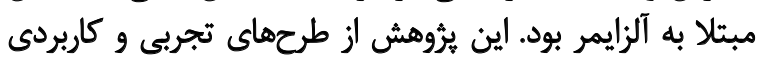

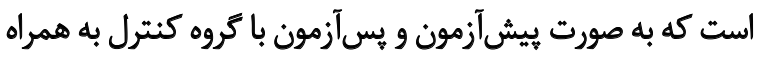

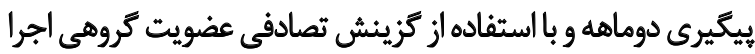

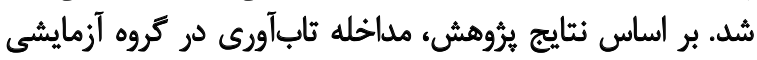

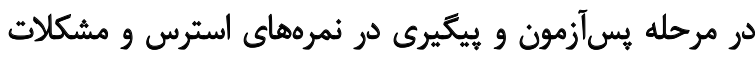

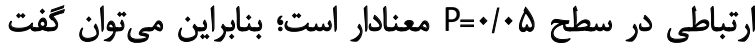

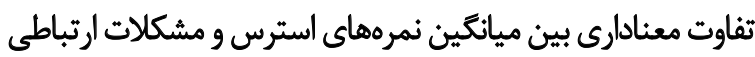
در يسآزمون و ئيكيرى وجود دارد. نتايج اين يُؤوش با نتايج مارتين كاراسكو و همكاران كه

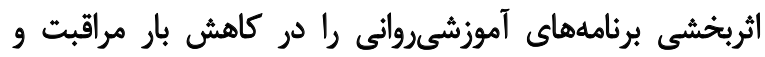

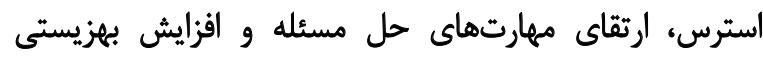

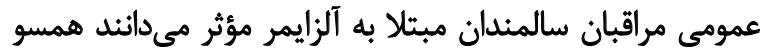

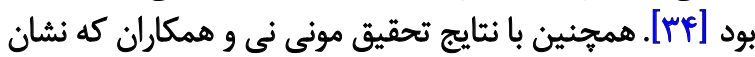

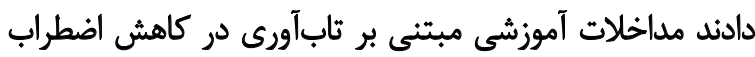

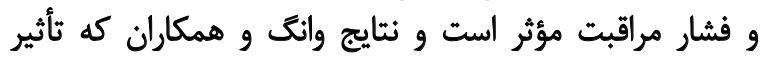

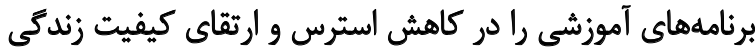

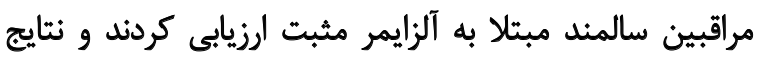

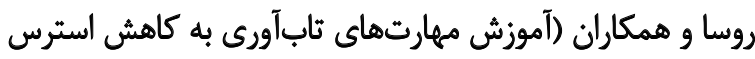

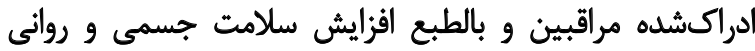

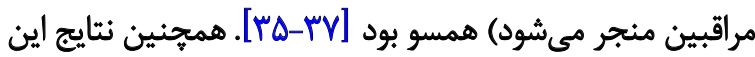

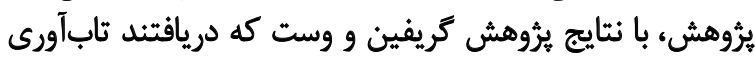


ضروريات آن مىشوند. آنها قادر به سازكارى سريع و شناخت

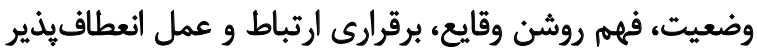

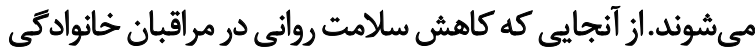

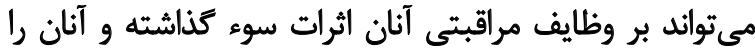

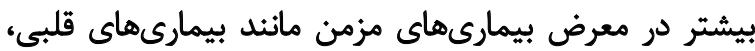

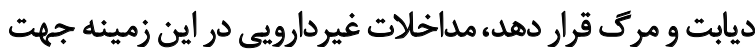

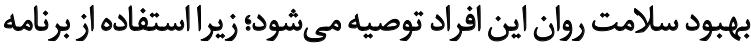

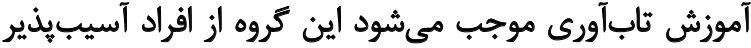

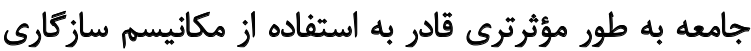

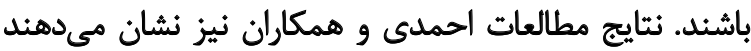

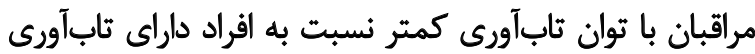

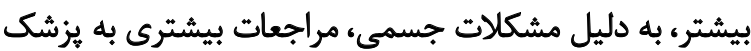

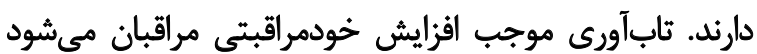

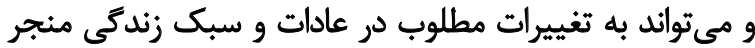

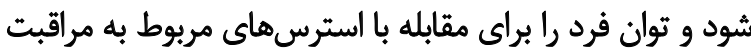

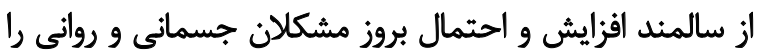

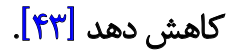

\section{نتيجه تيرى نمبايى}

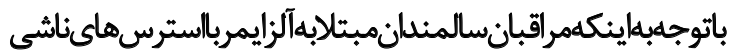

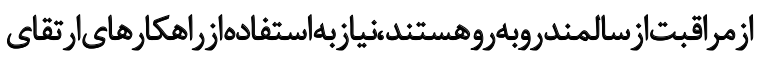

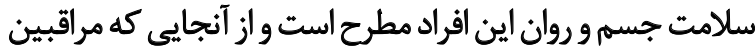

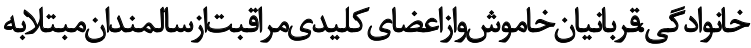

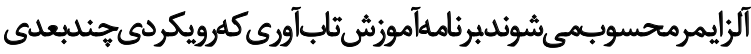

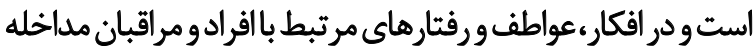

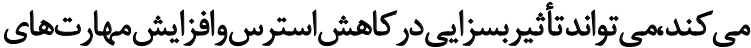

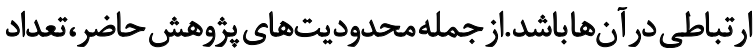

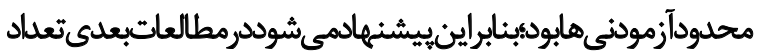

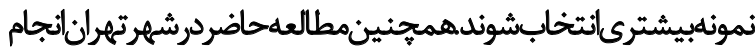

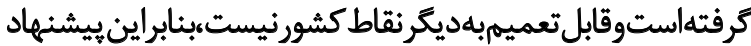
مىشود مطالعات مشابه در ساير مناطق كشور انجام شود.

مالاحظات اخلاقى

\section{يبيروى از اصول اخلاق يثوهش}

يثوهش حاضر در كميته يثوهشى دانشكاه كنبدكاووس و

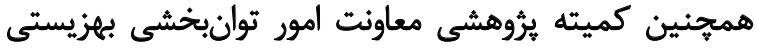
استان تهران به تأييد رسيده است.

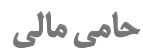

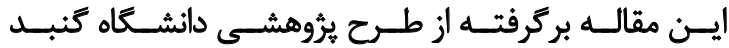

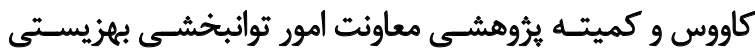

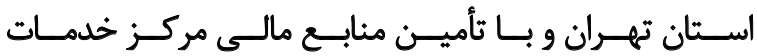

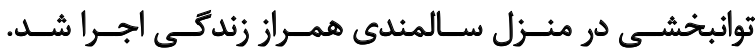

مكانيسمهاى سازكارى و ارائه مراقبت بهتر از سالمند هستند. به

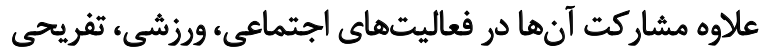

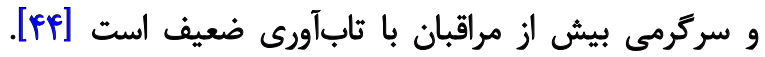

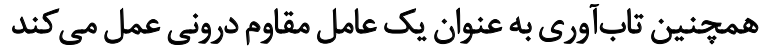

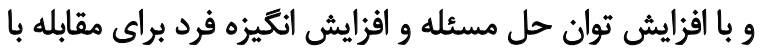

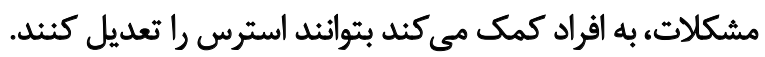

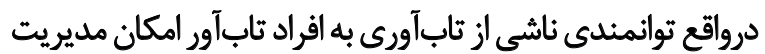

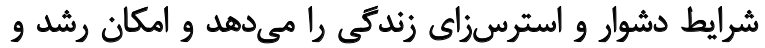

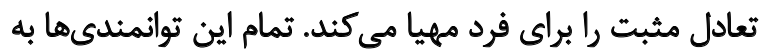

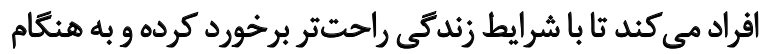

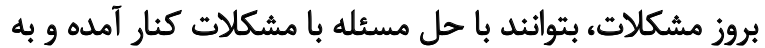
آرامش درونى بيشترى برسند [اس].

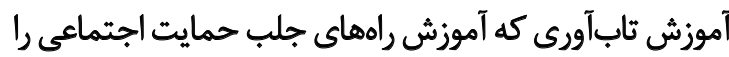

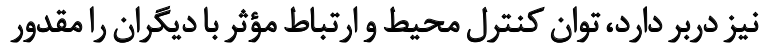

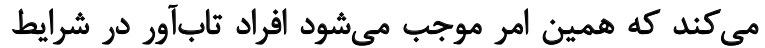

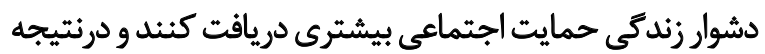

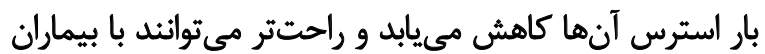

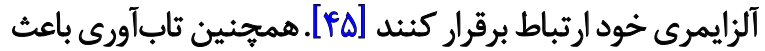

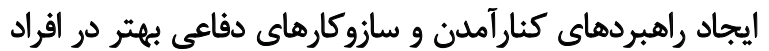

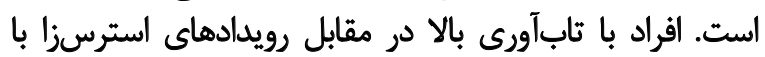

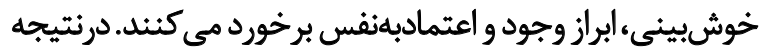

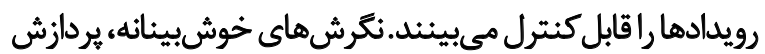

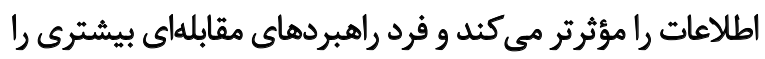

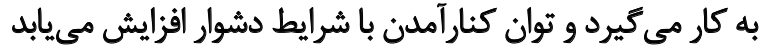

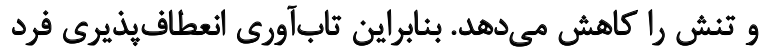

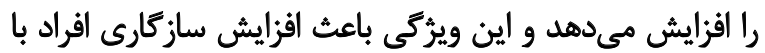

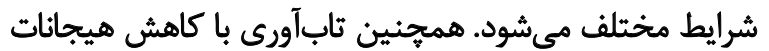

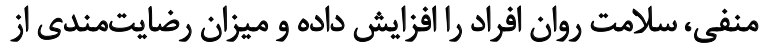

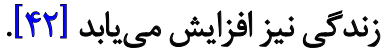
طبق نظر لويريزى و همكاران، توانايى حل كردن مشكلات،

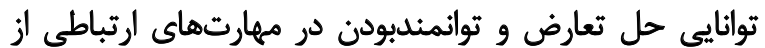

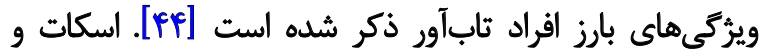

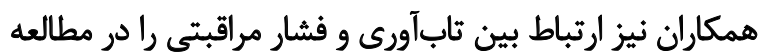

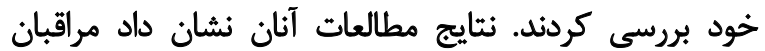

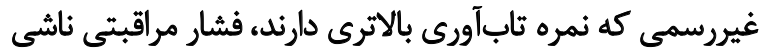

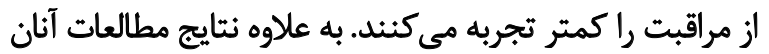

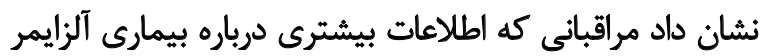

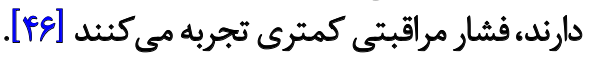

طبق نظر دنت توانايى حل كردن مشكلات و مهارتهاتي حل

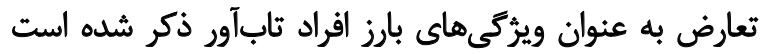

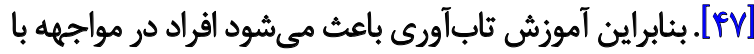

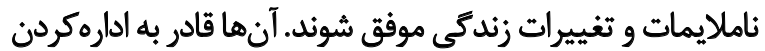

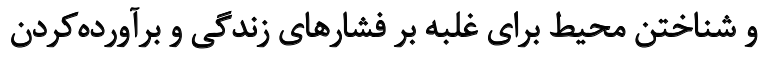




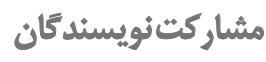

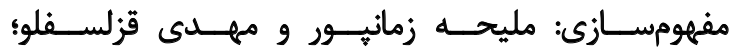

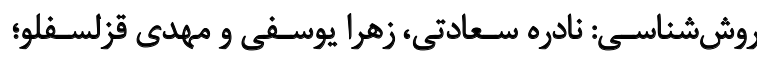

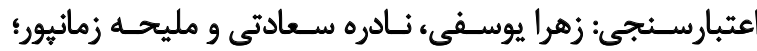

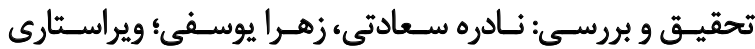

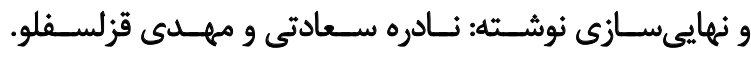

$$
\text { ế }
$$

بنابر اظهار نويسندكان الين مقاله تعارض منافع ندارد.. 


\section{References}

[1] Sadri Damirchi E, Ghomi M, Esmaeli Ghazi Valoii F. [Effectiveness of life review group therapy on psychological well-being and the life expectancy of elderly women (Persian)]. Iranian Journal of Ageing. 2017; 12(3):312-25. [DOI:10.21859/sija.12.3.312]

[2] Zamani SN, Bahrainian SA, Ashrafi S, Moqtaderi SH. [Effect of spiritual intelligence on quality of life and psychological well being among elderly living at nursing homes in Bandar Abbas (Persian)]. Journal of Geriatric Nursing. 2015; 1(4):82-94.

[3] Signe A, Elmståhl S. Psychosocial intervention for family caregivers of people with dementia reduces caregiver's burden: Development and effect after 6 and 12 months. Scandinavian Journal of Caring Sciences. 2008; 22(1):98-109. [DOI:10.1111/ j.1471-6712.2007.00498.x] [PMID]

[4] Moniz-Cook E, Vernooij-Dassen M, Woods B, Orrell M, Network I. Psychosocial interventions indementia care research: The INTERDEM manifesto. Aging and Mental Health. 2011; 15(3):283-90. [DOI:10.1080/13607863.2010.543665] [PMID]

[5] Egan M, Bérubé D, Racine G, Leonard C, Rochon E. Methods to enhance verbal communication between individuals with Alzheimer's disease and their formal and informal caregivers: A systematic review. International Journal of Alzheimer's Disease. 2010; 2010(906818):1-12. [DOI:10.4061/2010/906818] [PMID] [PMCID]

[6] Krishnamurthy V, Issac NS, Natarajan J. Computational identification of Alzheimer's disease specific transcription factors using microarray gene expression data. Journal of Proteomics \& Bioinformatics. 2009; 2(12):505-8. [DOI:10.4172/jpb.1000113]

[7] Chaudhuri JD, Das S. The role of caregivers in the management of Alzheimer's disease: Examples from Asian Countries. Sultan Qaboos University Medical Journal. 2006; 6(2):11-8. [PMID] [PMCID]

[8] Thies W, Bleiler L.Alzheimer's disease facts and figures. Alzheimer's \& Dementia. 2011; 7(2):208-44. [DOI:10.1016/j. jalz.2011.02.004] [PMID]

[9] Bastani F, Hosseini RS, Javanbakhtian Ghahfarokhi R. [The effect of group discussion and telephone counselling on perceived stress of women as caregivers of patient with Alzheimer disease (Persian)]. Journal of Clinical Nursing and Midwifery. 2012; 1 (1):61-72.

[10] Yektatalab S, Sharif F, Petramfar P. [Caring for patients with Alzheimer's disease in nursing homes: A qualitative content analysis (Persian)]. Journal of Qualitative Research in Health Sciences. 2012; 1(3):240-53. [DOI:10.1016/S0924-9338(12)74631-X]

[11] Khatooni M, Zohari S. A. [Survey on communicative problems between elders with Alzheimer disease and their family care givers (Persian)]. Iranian Journal of Ageing. 2010; 5(3):36-42.

[12] Mohammadi F, Fallahi Khoshknab M, Khanakeh H. [Caregivers 'needs for Alzheimer's elderly: An analysis of family corers' experience (Persian)]. Journal of Nursing and Midwifery Faculty.2011; 21(72):29-36.

[13] Bastani F, Ghasemi E, Negarandeh R, Haghani H. [General selfefficacy among family's female caregiver of elderly with Alzheimer's disease (Persian)]. Hayat. 2012; 18(2):27-37.

[14] Abdollahpour I, Noroozian M, NedjatS, Majdzadeh R. [Psychiatric symptoms in patients with dementia: Prevalence and their relationship with caregiver burden (Persian)]. Iranian Journal of Epidemiology. 2011; 7(2):51-9.
[15] Brodaty H, Donkin M. Family caregivers of people with dementia. Dialogues in Clinical Neuroscience. 2009; 11(2):217-28.

[16] Brodaty H, Green A, Koschera A. Meta-analysis of psychosocial interventions for caregivers of people with dementia. Journal of the American Geriatrics Society. 2003; 51(5):657-64. [DOI:10.1034/ j.1600-0579.2003.00210.x] [PMID]

[17] Mansouri T, Dehdari T, Seyedfatemi N, Gohari MR, Barkhordari $\mathrm{M}$. [The effect of communication skills training on perceived stress of caregivers of elderly with Alzheimer's disease referral to Iranian Alzheimer Association in 2012 (Persian)]. Razi Journal of Medical Sciences. 2014; 21(121):52-62.

[18] Etters L, Goodall D, Harrison BE. Caregiver burden among dementia patient caregivers: A review of the literature. Journal of the American Association of Nurse Practitioners. 2008; 20(8):423 8. [DOI:10.1111/j.1745-7599.2008.00342.x] [PMID]

[19] Farran CJ, Loukissa D, Perraud S, Paun O. Alzheimer's disease caregiving information and skills. Part II: Family caregiver issues and concerns. Research in Nursing \& Health. 2004; 27(1):40-51. [DOI:10.1002/nur.20006] [PMID]

[20] Savundranayagam MY, Hummert ML, Montgomery RJ. Investigating the effects of communication problems on caregiver burden. The Journals of Gerontology Series B. 2005; 60(1):S48-55 [DOI:10.1093/geronb/60.1.S48] [PMID]

[21] Broughton M, Smith ER, Baker R, Angwin AJ, Pachana NA Copland DA, et al. Evaluation of a caregiver education program to support memory and communication in dementia: A controlled pre-test-post-test study with nursing home staff. International Journal of Nursing Studies. 2011; 48(11):1436-44. [DOI:10.1016/j. ijnurstu.2011.05.007] [PMID]

[22] Millán-Calenti JC, Gandoy-Crego M, Antelo-Martelo M, LópezMartinez M, Riveiro-López MP, Mayán-Santos JM. Helping the family carers of Alzheimer's patients: From theory to practice. A preliminary study. Archives of Gerontology and Geriatrics. 2000; 30(2):131-8. [DOI:10.1016/S0167-4943(00)00044-3]

[23] Masten AS. Ordinary magic: Resilience processes in development. American Psychologist. 2001; 56(3):227-38. [DOI:10.1037// 0003-066X.56.3.227] [PMID]

[24] Kordich Hall D, Pearson J. Resilience - giving children the skills to bounce back. Education and Health. 2005; 23(1):2-15.

[25] Connor KM, Davidson JR. Development of a new resilience scale: The Connor-Davidson Resilience Scale (CD-RISC). Depression and Anxiety. 2003; 18(2):76-82. [DOI:10.1002/da.10113] [PMID]

[26] NooneSJ, Hastings RP. Building psychological resilience in support staff caring for people with intellectual disabilities: Pilot evaluation of an acceptance-based intervention. Journal of Intellectual Disabilities. 2009; 13(1):43-53. [DOI:10.1177/1744629509103519] [PMID]

[27] Friborg $\mathrm{O}$, Hjemdal $\mathrm{O}$, Rosenvinge $\mathrm{JH}$, Martinussen $\mathrm{M}$, Aslaksen PM, Flaten MA. Resilience as a moderator of pain and stress. Journal of Psychosomatic Research. 2006; 61(2):213-9. [DOI:10.1016/j.jpsychores.2005.12.007] [PMID]

[28] Jennings DA. [The effects of resiliency training on physical activity participation, job satisfaction, and protective factors among nonmanagerial females at the worksite [PhD. dissertation]. Utah: University of Utah; 2002. 
[29] Momeni K, Karimi H. [Comparison of mental health between elderly admitted in sanitarium with elderly in sited in personal home (Persian)]. Journal of Kermanshah University of Medical Sciences. 2011; 14(4):328-35.

[30] Shareh H. [The effect of work stress, coping strategies, resilience and mental health on job satisfaction among anesthesia technicians (Persian)]. Journal of Fundamentals of Mental Health. 2011; 13(1):20-9.

[31] Hosseni. T, Salimi. M. [The effect of resilience training on the stress of mothers with cancer children in Imam Khomeini Hospital (Persian)]. Health Psychology Journal. 2012; 1(4):97-109.

[32] Jadidian A, Solgi M. [Effectiveness of resilience training on reduction of parental stress of autistic childrens mothers (Persian)]. Scientific Journal of Ilam University of Medical Sciences. 2015; 23(4):95-105.

[33] Cohen S, Kamarck T, Mermelstein R. A global measure of perceived stress. Journal of Health and Social Behavior. 1983; 24:35896. [DOI:10.2307/2136404] [PMID]

[34] Hashemi T, Peymannia B. [The relationship between (D) type of personality, and perceived stress with health behaviors in women with breast cancer (Persian)]. International Journal of Palliative Nursing. 2014; 1(4):36-44.

[35] Martín-Carrasco M, Martín MF, Valero CP, Millán PR, García CI, Montalbán SR, et al. Effectiveness of a psychoeducational intervention program in the reduction of caregiver burden in Alzheimer's disease patients' caregivers. International Journal of Geriatric Psychiatry. 2009; 24(5):489-99. [DOI:10.1002/gps.2142] [PMID]

[36] Monini P, Tognetti A, Cinque R, Di Franco F, Bartorelli L. [The importance of awareness: An experience of small support groups for the caregivers of Alzheimer's disease patients (Persian)]. Archives of Gerontology and Geriatrics. 2001; 1(33):267-71. [DOI:10.1016/S0167-4943(01)00148-0]

[37] Wang JJ, Hsu YC, Cheng SF. The effects of reminiscence in promoting mental health of Taiwanese elderly. International Journal of Nursing Studies. 2005; 42(1):31-6. [DOI:10.1016/j. ijnurstu.2004.05.010] [PMID]

[38] Rosa E, Lussignoli G, Sabbatini F, Chiappa A, Di Cesare S, Lamanna L, et al. Needs of caregivers of the patients with dementia. Archives of Gerontology and Geriatrics. 2010; 51(1):54-8. [DOI:10.1016/j.archger.2009.07.008] [PMID]

[39] Griffith J, West C. Master resilience training and its relationship to individual well-being and stress buffering among army national guard soldiers. The Journal of Behavioral Health Services \& Research. 2013; 40(2):140-55. [DOI:10.1007/s11414-013-9320-8] [PMID]

[40] Xin Z, Li P, Jinsheng W, Min L, Minqin Z, Anqiang Y, et al Effect of resilience training onself-awareness and coping style of warship soldiers. Journal of Third Military Medical University. 2013; 2013(15):24.

[41] Bazzazian S, Rajabi A. [Self-efficacy, social support and quality of life in patients with type II diabetes (Persian)]. Journal of Behavioral Science. 2011; 3(10):25-39.

[42] Smith JM, Alloy LB. A roadmap to rumination: A review of the definition, assessment, and conceptualization of this multifaceted construct. Clinical Psychology Review. 2009; 29(2):116-28. [DOI:10.1016/j.cpr.2008.10.003] [PMID] [PMCID]
[43] Ahmadi R, Sharifi Darmandi P. [A studyof the effect of resilience training on mental health of people with drug dependency at Touska Camp in Tehran (Persian)]. Quarterly of Clinical Psychology Studies. 2014; 4(16):1-17.

[44] Mohammadi Shahbolaghi F. [Selfefficacyand burden of caregiver's asfamily members of elderly with Alzheimer disease in Tehran (Persian)]. Iranian Journal of Ageing. 2006; 6(1):26-33.

[45] Loprinzi CE, Prasad K, Schroeder DR, Sood A. Stress Management and Resilience Training (SMART) program to decrease stress and enhance resilience among breast cancer survivors: A pilot randomized clinical trial. Clinical Breast Cancer. 2011 11(6):364-8. [DOI:10.1016/j.clbc.2011.06.008] [PMID]

[46] Scott CB. Alzheimer's disease caregiver burden: Does resilience matter? Journal of Human Behavior in the Social Environment. 2013; 23(8):879-92. [DOI:10.1080/10911359.2013.803451]

[47] Dent M. Stop stealing childhood in the name of education: A plea to ask WHY? Maryland: Office of Federal Education Minister; 2013. 
This Page Intentionally Left Blank 